\title{
Design on controllers for electrical appliances in smart home
}

\author{
Tran Que Son, Tran Manh Tuan, Le Van Hoan, Phung Thanh Hai, Nguyen \\ Minh $\mathrm{Y}^{*}$ \\ Faculty of International Training, Thai Nguyen Univresity of Technology, 3-2 St. Tich Luong dist. Thai Nguyen, Vietnam
}

\begin{abstract}
This paper presents a theoretical and practical design of controllers for electrical appliances in smart home. Taking into account the advantage of information and automation technologies, the home appliance can be controlled accordingly to the price signal of electricity to minimize the total energy cost over a period of time. The problem is formulated in Dynamic Programming framework and solved by Dynamic Program backward algorithm. Then, the proposed scheme is tested in case study to control the charging process of Electric Vehicle; a comparison to the traditional scheme is performed to illustrate its effectiveness. Currently, a "testbed" model of smart home is under construction at Thai Nguyen University of Technology in which the proposed scheme will be applied to control the typical home appliances with a communication system based on Zigbee protocol.
\end{abstract}

Keywords: Demand response; smart grid; smart home; electric vehicle

\section{Introduction}

The ability to adjust or cut off the electric consumption of home appliance is referred to as Demand Response (DR) program, for instance turning off the appliance at peak times to alleviate the need to pay an expensive electricity cost. It is thanks to Smartgrid technologies with communication and automation applied to the electric grid, which enables the information to be exchanged in real-time and makes DR even more possible and become an important resource in the power system [1]. With the deregulation of the electric power industry and the implementation of electricity markets, the benefits of DR are shared between system operators and electric users. The user can save the electricity bill by actively responding to the price signal, by this means the balance of power supply and demand in the whole system can be guaranteed [2].

Generally, electrical appliances can be categorized into three groups: (1) sheddable loads, appliances that, when turning on/off would be slightly (or un-) noticed by the users (e.g., water heater); (2) shiftable loads, appliances with a flexible starting time that only needs to be accomplished by a specified time (e.g., washing machine); and (3) critical loads, appliances that, when turning on/off could result in some inconvenience or discomfort for the users (e.g., lights, air-conditioning.)

There are many efforts have been paid to address the DR problem for different kind of home appliances under diverse scenarios. The scheduling of ILs, i.e., the portion and duration of interruptions, as a part of the optimal generation is presented in [3]. In [4], the multi-objective optimization is employed to take care of conflicting goals, such as minimizing payment to ILs, minimizing frequency of interruptions, etc. at the same time meeting the system requirements. DR is also considered upon the thermo-appliances of an aggregate load as a power source in [5] where the algorithm for short-term operations in electricity markets is presented. In [6], charging algorithm, namely "smart charger" for electric vehicles in Smargrids is proposed; it is concerned with the trade-off between the customers' willingness to pay and the charging rate.

*Manuscript received December 16, 2016; revised April 10, 2017.

Corresponding author. Tel.: +84966996399; E-mail address: minhy@tnut.edu.vn.

doi: $10.12720 /$ sgce.6.2.90-95 
In this paper, we present a theoretical and technical design of DR controller for home appliances in Smartgrid. The aim of the controller is to find out the optimal operation of each appliance over a predetermined period with minimum energy cost. Analyzing the technical property of different kinds of appliances and also the user's requirement, the DR controller is able to adjust or turn on/off the home appliance to reduce the cost but does not affect the comfort level of users. The proposed control scheme consists of two functions: (1) Ahead scheduling based on the anticipated data and (2) real-time control following both the scheduling and the measurement or updated information of the appliance. The problem is formulated in Dynamic Programming (DP) framework and solved by DP Backward Algorithm (DPBA). The simulation results in Case Study show the capability and performance of the proposed DR controller and a comparison to the traditional control scheme is done to highlight its effectiveness. Further, a "testbed" model of "smart home" was under construction at Thai Nguyen University of Technology with the technical design of the proposed DR controller.

The remainder of the paper is organized as follows. Section II presents the mathematical formulation of DR problem for the charger of Electric Vehicle (EV). Section III introduces the solution algorithm by DPBA. Section IV presents an illustrative example where the proposed DR controller is applied to schedule and operate the charging process of EV. Section V is Conclusion where the significant points and remarks of the paper are summarized.

\section{Mathematical Formulation}

Under market environments, electric users are offered choices to pay their usage corresponding to the condition of the wholesale market, i.e., real-time price. The problem is to schedule ahead of time for the operation of electrical appliances to minimize the energy cost over a predetermined period. The mathematical formulation of DR problem for EVs is as follows.

$$
\min _{\substack{q_{k}^{v v} \\ k=0, \ldots N-1}} \sum_{k=0}^{N-1} \rho_{k} q_{k}
$$

Subject to

$$
\begin{aligned}
& \operatorname{soc}_{k+1}= \begin{cases}s o c_{k}+\eta q_{k} & \text { if charging } \\
s o c_{k}-\frac{1}{\eta} q_{k} & \text { if discharging }, k=0,1 \ldots N-1\end{cases} \\
& q_{\text {min }} \leq q_{k} \leq q_{\text {max }}, k=0,1 \ldots N-1 \\
& s o c_{\text {min }} \leq \operatorname{soc}_{k} \leq s o c_{\text {max }}, k=1,2 \ldots N \\
& \operatorname{soc}_{N}=\operatorname{soc}_{\max }
\end{aligned}
$$

where, $\mathrm{q}_{\mathrm{k}}$ is the energy charging/discharging of the EV during stage $\mathrm{k},[\mathrm{kWh}]$; $\operatorname{soc}_{\mathrm{k}}$ is the battery SOC at the beginning of stage $\mathrm{k},\left[\mathrm{kWh}\right.$ ]; $\eta$ is the charging/discharging efficiency, [p.u.]; $\mathrm{q}_{\min }, \mathrm{q}_{\max }$ are the charging/discharging rate limits, $[\mathrm{kW}]$; and $\operatorname{soc}_{\min }$, $\operatorname{soc}_{\max }$ are the capability limits of the battery bank, $[\mathrm{kWh}]$. It is noted that the number of stage, $\mathrm{N}$, just covers a portion of day i.e., from the time when the EV is plugged to the charger to the time it needs to be used (e.g., 06:00PM-08:00AM). The user's preference is that the EV is full of charge at the end of charging duration (Eq. 5).

\section{Dynamic Programming}

DP is an effective method for many problems in electrical engineering, particularly to deal with the 
uncertainty, nonlinearity and inter-correlation effects (i.e., the current decision will affect the optimality of the later process) of the problems [7]. The method is to define the control policy, a set of functions of the system state that would point out the optimal control of the system in real-time operation given the current state (i.e., after the uncertainty is cleared). The block diagram of DP and its variables defined in time scale are presented in Fig. 1.

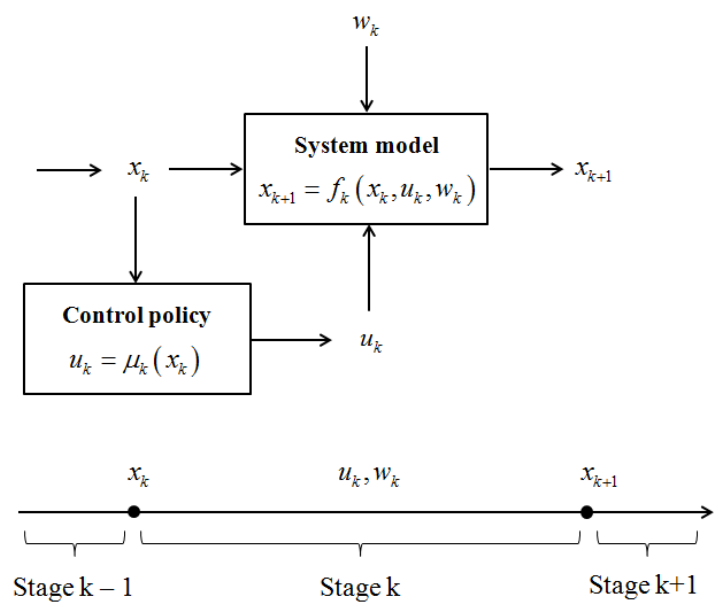

Fig. 1. The block diagram of DP and its variable in time scale

In Fig. 1, let $\mathrm{x}_{\mathrm{k}}$ be the system state variable that indicates the SOC of battery at the beginning of stage $\mathrm{k}$; $\mathrm{u}_{\mathrm{k}}$ be the control variable that indicates the amount of charging/discharging to the battery during stage $\mathrm{k} ; \mathrm{w}_{\mathrm{k}}$ be the uncertainty in the stage $\mathrm{k}$. The DPBA approach starts from the last stage and gradually moves backward to first one, i.e., namely backward algorithm. In each stage, the sub-problems, to search for the optimal control of the following stage given the current state are solved; also, a performance index called cost-to-go function is determined which indicates the minimum added cost of the entire following stages. Hence, it can be realized that the manipulation in each stage provides information of how best the performance can be in future (i.e., the remaining stages.) The optimal control at each stage is archived to assemble the control policy, $\mu_{\mathrm{k}}\left(\mathrm{x}_{\mathrm{k}}\right)$, as aforementioned. The algorithm terminates when the initial stage is reached and the control policy of all possible states is defined. The DPBA at stage $\mathrm{k}$ can be described mathematically as follows.

$$
\begin{aligned}
& J_{k}\left(x_{k}\right)=\min _{u_{k}} \underset{w_{k}}{E}\left\{J_{k+1}\left(f_{k}\left(x_{k}, u_{k}, w_{k}\right)\right)+g_{k}\left(x_{k}, u_{k}, w_{k}\right)\right\} \\
& \mu_{k}\left(x_{k}\right)=u_{k}^{*}
\end{aligned}
$$

where, $\mathrm{g}_{\mathrm{k}}\left(\mathrm{x}_{\mathrm{k}}, \mathrm{u}_{\mathrm{k}}, \mathrm{w}_{\mathrm{k}}\right)$ is the cost function that indicates the cost associated with the current decision (Eq. 1); $\mathrm{f}_{\mathrm{k}}\left(\mathrm{x}_{\mathrm{k}}, \mathrm{u}_{\mathrm{k}}, \mathrm{w}_{\mathrm{k}}\right)$ is called the state transition function that indicates how the system state changes according to the current decision (Eq. 2); $\mathrm{u}_{\mathrm{k}}{ }^{*}$ is the solution of the optimization problem in (Eq. 6) and (Eq. 7). It is noted that due to the ending constraint, the value of cost-to-go function is set to be zero at the last stage with the ending state, otherwise be infinite. It is the same with the starting constraint. With the control policy determined ahead of time, the decision in real-time operation can be made as simply as:

$$
u_{k}^{*}=\mu_{k}\left(x_{k}\right)
$$

\section{Case Study}

In this section, the proposed DR scheme is applied to control the charging process of EVs. The 
problem is that when the user arrives home (for example after work at 18:00 PM today), the EV is plugged to the power charger with the desire that the EV to be full-of-charge after certain duration (e.g., to go for work at 08:00 AM tomorrow). Traditionally, the EV with be charged with a fixed rate set by the user until full as follows.

$$
q_{k}= \begin{cases}q_{r e f} & \text { if } \quad \operatorname{soc}_{k}<s o c_{\max } \\ 0 & \text { otherwise }\end{cases}
$$

where, $\mathrm{q}_{\mathrm{k}}$ is the charging rate at stage $\mathrm{k},[\mathrm{kW}]$; $\mathrm{q}_{\mathrm{ref}}$ is the reference charging rate, $[\mathrm{kW}]$. Differently, with the proposed DR scheme, the charger need to update and/or estimate the electricity price over the charging period based on the historical data. After that the scheduling algorithm will be performed to determine the control policy of the charging process with respect to the uncertainty of electricity price. Then, in real-time, the controller will follow the control policy and its measurement of the system state to control the charging process (time and amount of charging).

The price signal used in this study is obtained by modifying the hourly electricity price of PJM market [8]. It is worth noted that the hourly price is determined ahead of time through a bidding process in electricity markets, i.e., scheduling for anticipated loads. The imbalance in real-time operation caused by load deviation from the anticipated will be handled by calling upon regulation services (up or down); this action results in the real-time price different from the hourly price [9]. The price signal is presented in Fig. 2 .

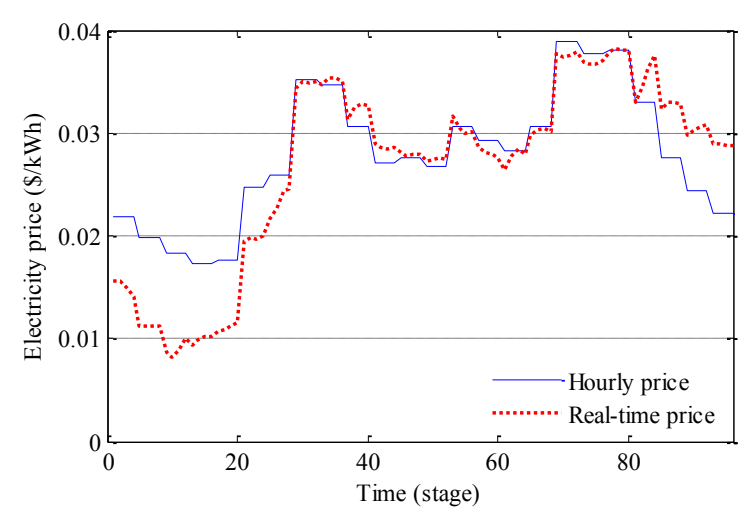

Fig. 2. The electricity price: Hourly price and real-time price, $[\$ / \mathrm{kWh}]$.

The detail formulation and parameters needed in this simulation are presented Table 1.

Table 1. Parameter of the charging scheme for EV

\begin{tabular}{lll}
\hline EV's parameters & Value & Unit \\
\hline$\eta$ & 0.85 & p.u. \\
$\operatorname{soc}_{\max }$ & 50 & $\mathrm{kWh}$ \\
$\operatorname{soc}_{\min }$ & 10 & $\mathrm{kWh}$ \\
$\mathrm{q}_{\min }$ & 0 & $\mathrm{~kW}$ \\
$\mathrm{q}_{\max }$ & 5 & $\mathrm{~kW}$ \\
\hline
\end{tabular}

The DR problem of EVs is not just charging at off-peak hours but capable of providing power back to the grid for profits; this idea also can be referred to as Vehicle-to-Grid (V2G) [10]. The operation scheme of Battery Energy Storage (BES) in response to the real-time and frequency control markets is addressed in [9] and [10], respectively. The problem here is a little different that the EV is only plugged into the grid in a certain period, e.g., 06:00 PM-08:00 AM in this study and must be full of charge at the end. The results of simulation are presented in Fig. 3. 

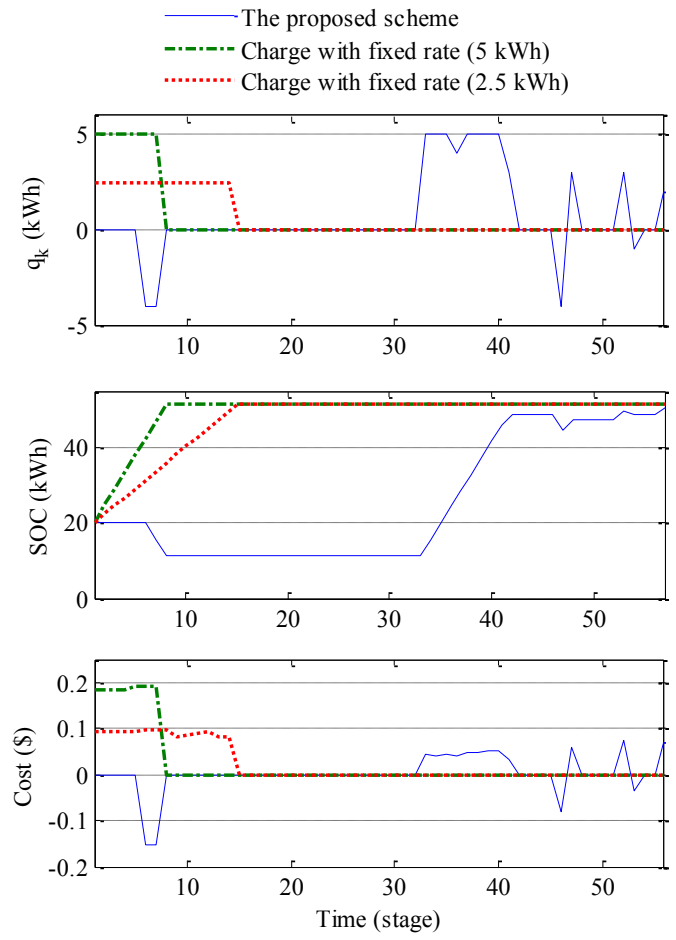

Fig. 3. The charging/discharging amount, [kWh], battery SOC, $[\mathrm{kWh}]$, and electricity cost, $[\$]$.

As can be seen from Fig. 3, the proposed DR scheme intents to discharge the battery when the price of electricity is high (from 07:15 PM to 07:45 PM) and charge when the price is low (from 01:30 AM to 03:45 AM) and so on. It is different from the traditional scheme where the EV is charged with a fixed rate, the battery is charged constantly until full. This will result in pretty higher cost $\left(1.31 \$\right.$ with $\mathrm{q}_{\mathrm{ref}}=5 \mathrm{~kW}$ and $1.27 \$$ with $\left.\mathrm{q}_{\mathrm{ref}}=2.5 \mathrm{~kW}\right)$ compared to that of the proposed scheme $(0.49 \$)$. The saving is huge in this case.

\section{Conclusion}

In this paper, we have proposed a new DR control scheme for EVs in electricity markets using DP framework and the solution by DPBA. The objective function is to minimize the total energy cost of EVs over the predetermined period while satisfy the user's preference. The proposed scheme consists of two parts: (1) Scheduling ahead of time based on the anticipated data and (2) real-time control following both the ahead scheduling and the measurement or updated information. In case study, the proposed scheme is applied to control the charging/discharging process of EVs givens the price from the electricity market. The result showed that significant reduction of the cost can be obtained by discharging the battery in the high price time while charging in the low price time. As future work, we will apply this scheme and build a "testbed" model of smart home where all the electrical appliances are communicated and controlled in real-time as proposed in this paper.

\section{Acknowledgement}

This work is supported by Thai Nguyen University of Technology as parts of its project of Smartmeter and Demand-side Management Systems. 


\section{References}

[1] Chowdhury S, Chowdhury SP. Microgrids and active distribution network. In: The Institution of Engineering and Technology, London, United Kingdom, 2009.

[2] Balijepalli VS, Pradhan V, Khaparde SA, Shereef RM. Review of demand response under smart grid paradigm. In: Proc. Innovative Smart Grid Technologies, Keraka, India, 2011:1-8.

[3] Brooks A, Lu E, Reicher D, Spirakis C, Weihl B. Demand dispatch: Using real-time control of demand to help balance generation and load. IEEE Power and Energy Magazine, 2010; 5(3):20-29.

[4] Majumdar S, Chattopadhyay D, Parikh J. Interruptible load management using optimal power flow analysis. IEEE Trans. Power Systems, 1996; 11(2):715-720.

[5] Pedrasa MA, Spooner TD, MacGill IF. Scheduling of demand side resources using binary particle swarm optimization. IEEE Trans. Power Systems, 2006; 24(3):1173-1181.

[6] Ruiz N, Cobelo I, Oyazabal J. A direct load control model for virtual power plant manegements. IEEE Trans. Power Systems, 2009; 24(2):959-966.

[7] Bertsekas DP. Dynamic Programming and Optimal Control. Athena Scientific: Belmont, MA, USA, 1995.

[8] PJM Electricity market. [Online]. Availale: http://www.pjm.com

[9] Nguyen MY, Nguyen VT, Yoon YT. A new battery energy storage charging/discharging scheme for wind power producers in real-time markets. Energies, 2012; 5(12):5439-5452.

[10] Ota Y, Taniguchi H, Nakajima T, Liyanage K, Baba J, Yokoyama A. Autonomous distributed V2G (Vehicle-to-Grid) satisfying scheduled charging. IEEE Trans. Smart Grid, 2014; 3(1):559-565. 